\title{
Outcomes of neoadjuvant chemoradiotherapy followed by total mesorectal excision surgery for locally advanced rectal cancer: a single-institution experience
}

Michelle Shu Fen Tseng ${ }^{1}$, MBBS, Huili Zheng ${ }^{2}$, BSc, Ivy Wei Shan Ng$^{1}$, MBBS, MRCP, Yiat Horng Leong ${ }^{1}$, MBBS, FRANZCR, Cheng Nang Leong ${ }^{1}$, MBBS, FRAnZCR, Wei Peng Yong ${ }^{3}$, MB ChB, MRCP, Wai Kit Cheong ${ }^{4}$, MBBS, FRCS, Jeremy Chee Seong Tey $^{1}$, MBBS, FRANZCR

INTRODUCTION Neoadjuvant chemoradiotherapy (CRT) followed by total mesorectal excision (TME) surgery for locally advanced rectal cancer has been shown to improve local control and reduce toxicity, as compared to adjuvant CRT. We reported the outcomes of our patients with locally advanced rectal cancer treated at National University Hospital, Singapore. METHODS From April 2002 to December 2014, 117 patients with T3/4, N0/+, M0 rectal cancer received neoadjuvant CRT followed by TME surgery. The treatment regimen comprised a total radiotherapy dose of 50.4 Gy in 28 daily fractions delivered concurrently with 5-fluorouracil or capecitabine chemotherapy over 5.5 weeks. All patients were planned for TME surgery. Local control, disease-free survival, overall survival and treatment toxicities were analysed.

RESULTS Median follow-up was 34 (range 2-122) months. 11.5\% (13/113) of patients achieved a pathological complete response $(\mathrm{pCR})$ and $72.6 \%$ (85/117) had either tumour or nodal downstaging following neoadjuvant CRT. 5.2\% (5/96) of patients had Grade 3 acute toxicities (dermatitis and diarrhoea) and 3.1\% (3/96) had Grade 3 late toxicities (fistula and stricture). There was no Grade 4 toxicity noted. The five-year local recurrence, disease-free survival and overall survival rates were $4.5 \%, 65.7 \%$ and $80.6 \%$, respectively. Multivariate analysis showed that nodal positivity was a predictor of poor disease-free survival and poor overall survival. Tumour downstaging and pCR did not improve outcomes. CONCLUSION Our outcomes were comparable to internationally published data, and this treatment regimen remains the standard of care for locally advanced rectal cancer in our local population.

Keywords: chemotherapy, neoadjuvant therapy, radiotherapy, rectal cancer, surgery

\section{INTRODUCTION}

Colorectal cancer remains one of the most common cancers in both men and women in Singapore, with the ethnic Chinese population at a higher risk than the Malay and Indian populations. Incidence rates in Singapore have increased since the 1970s but have plateaued since the late 1990s. Mortality rates from colorectal cancer are among the highest in both genders. ${ }^{(1)}$

Several important historical trials helped to establish trimodality therapy as the standard treatment for locally advanced rectal cancer nearly 20 years ago. ${ }^{(2,3)}$ The results of a large, randomised controlled trial comparing preoperative chemoradiation against postoperative chemoradiation have demonstrated improved local control and reduced toxicities with a neoadjuvant approach. ${ }^{(4,5)}$ Neoadjuvant chemoradiotherapy (CRT) for locally advanced rectal cancer was implemented at our institution in 2002. In this study, we reported the outcomes of our patients with locally advanced rectal cancer over a 12-year period.

\section{METHODS}

Eligible patients were those who were diagnosed with clinically staged T3/4, NO/+, MO rectal cancer according to the TNM (tumour, node and metastasis) Classification of Malignant Tumours and received neoadjuvant CRT followed by total mesorectal excision (TME) surgery at National University Hospital, Singapore, from April 2002 to December 2014. Patients were selected from the Radiation Oncology Department's internal database based on diagnosis coding and total radiotherapy dose delivered. Additional information was collected from both internal database reporting and hospital electronic medical records. All patients had biopsy-proven rectal adenocarcinoma.

All patients were investigated with magnetic resonance imaging, computed tomography (CT) of the pelvis or transrectal ultrasonography to determine tumour and nodal staging. Distant metastasis was excluded by CT imaging of the thorax and abdomen. Stage of disease was determined by the American Joint Committee on Cancer staging system (7th edition), which utilises the TNM scoring system. We defined low rectal tumours, mid rectal tumours and high rectal tumours as those that were less than $5 \mathrm{~cm}, 5-10 \mathrm{~cm}$ and more than $10 \mathrm{~cm}$ from the anal verge, respectively.

Chemotherapy was given as 5-fluorouracil (5-FU) intravenously or capecitabine orally. Overall, 40 patients received intravenous $5-\mathrm{FU}$ at a dose of $225 \mathrm{mg} / \mathrm{m}^{2} /$ day and the remaining 77 patients received oral capecitabine at a dose of $825 \mathrm{mg} / \mathrm{m}^{2}$ twice a day for five consecutive days a week when radiotherapy was administered. 
Radiotherapy was delivered as three-dimensional conformal radiotherapy with $6 \mathrm{MV}$ or $10 \mathrm{MV}$ photons. The beam arrangement consisted of either one posterior beam and two lateral beams, or one anterior and posterior beam and two lateral beams. The field borders, which were standard for all patients, were as follows: superior border was L5-S1 intervertebral space; inferior border was the inferior obturator foramen or $3 \mathrm{~cm}$ below the most inferior part of the tumour (whichever was more inferior); anterior border was $2-3 \mathrm{~cm}$ anterior to the sacral promontory; posterior border was $1 \mathrm{~cm}$ posterior to the sacrum; and lateral borders were $1-1.5 \mathrm{~cm}$ lateral to the pelvic brim. A total dose of 50.4 Gy in 28 fractions was delivered in two phases. Phase 1 delivered 45 Gy in 25 fractions to the pelvis as 1.8-Gy fractions over five consecutive weeks. Phase 2 gave a further tumour boost of 5.4 Gy in three daily fractions.

All patients underwent TME surgery, which included low anterior resection, ultra-low anterior resection, abdominoperineal resection or Hartmann's procedure. Surgery was planned 8-12 weeks after neoadjuvant CRT. Tumour size, nodal metastasis and margin status were identified from the patient's histology reports. Postoperative complications were also noted.

After completion of neoadjuvant CRT and TME surgery, all patients were followed up postoperatively with history taking, examination and carcinoembryonic antigen blood test every three months for the first two years, every six months during Years 3-5 and annually thereafter. $\mathrm{CT}$ of the chest, abdomen and pelvis were repeated annually to screen for local recurrence or distant metastasis. Postoperative colonoscopy was performed at Years 1, 3 and 5. This was in accordance with the National Comprehensive Cancer Network guidelines for surveillance. ${ }^{(6)}$

Radiation toxicities were recorded weekly during treatment reviews and at subsequent follow-up visits. The toxicities were scored using Common Terminology Criteria for Adverse Events version 4.0 (US Department of Health and Human Services, National Institute of Health, National Cancer Institute, MD, USA).

Kaplan-Meier curves were plotted to provide an overview of the local recurrence, disease-free survival and overall survival rates of all patients. Univariate and multivariate Cox regression analyses were done to determine the predictive factors for diseasefree survival and overall survival. Factors for which Cox regression analyses were performed included age at diagnosis, gender, chemotherapy regimen (intravenous 5-FU or oral capecitabine), margin positivity, tumour downstaging, node positivity and pathological complete response (pCR). All analyses were done using STATA SE version 13 (StataCorp LP, College Station, TX, USA). A p-value $<0.05$ was considered to be statistically significant.

\section{RESULTS}

During the study period, 127 patients had clinically staged cT3/4, $\mathrm{NO} /+$ adenocarcinoma of the rectum that was planned to be treated with neoadjuvant CRT followed by surgery. Four patients received cetuximab with 5-FU, five received intensity-modulated radiotherapy (IMRT) instead of conventional radiotherapy and one declined neoadjuvant CRT. These patients were excluded, leaving 117 patients who were eligible for analysis. $66.7 \%$ of the patients
Table I. Patient characteristics $(\mathrm{n}=117)$.

\begin{tabular}{|c|c|}
\hline Variable & No. (\%) \\
\hline \multicolumn{2}{|l|}{ Gender } \\
\hline Male & $78(66.7)$ \\
\hline Female & 39 (33.3) \\
\hline Age at diagnosis (yr)* & $60(24-81)$ \\
\hline \multicolumn{2}{|l|}{ Age group (yr) } \\
\hline$<70$ & $95(81.2)$ \\
\hline$\geq 70$ & $22(18.8)$ \\
\hline \multicolumn{2}{|l|}{ Ethnicity } \\
\hline Chinese & $84(71.8)$ \\
\hline Malay & $16(13.7)$ \\
\hline Indian & $13(11.1)$ \\
\hline Other & $4(3.4)$ \\
\hline \multicolumn{2}{|l|}{ ECOG performance status } \\
\hline 1 & $116(99.1)$ \\
\hline 2 & $1(0.9)$ \\
\hline \multicolumn{2}{|l|}{ Rectal tumour } \\
\hline Low & $53(45.3)$ \\
\hline Mid & $43(36.8)$ \\
\hline High & $12(10.3)$ \\
\hline Unknown & $9(7.7)$ \\
\hline \multicolumn{2}{|l|}{ Imaging for staging } \\
\hline CT & $34(29.1)$ \\
\hline TRUS & $4(3.4)$ \\
\hline CT and TRUS & $3(2.6)$ \\
\hline CT and MR imaging & $68(58.1)$ \\
\hline CT, TRUS and MR imaging & $1(0.9)$ \\
\hline None documented & $7(6.0)$ \\
\hline
\end{tabular}

*Data presented as median (range). CT: computed tomography; ECOG: Eastern Cooperative Oncology Group scale; MR: magnetic resonance; TRUS: transrectal ultrasonography

were men and the median age at diagnosis was 60 (range 24-81) years. $94(80.3 \%)$ of the patients were successfully followed up, and among these, the median follow-up time was 34 (range 2-122) months. The patients' characteristics are summarised in Table I.

Among patients who had surgery $(n=113)$, the median time from completion of neoadjuvant CRT to surgery was 90 (range 49-172) days. Of the 13 (11.5\%) patients who achieved a pCR, six had surgery at 8-12 weeks and seven had surgery after 12 weeks. Surgical procedures were decided preoperatively. Low anterior resections, ultra-low anterior resections, abdominoperineal resections and Hartmann's procedure were performed for 28, 61, 22 and two patients, respectively. Three patients declined surgery but had biopsies taken post neoadjuvant CRT, and one patient passed away before surgery due to acute myocardial infarction secondary to bleeding from the rectal tumour. The sphincter preservation rate was $79.6 \%$. TME was performed for all patients who had surgery. Negative resection margins were achieved in $91.2 \%$ of patients. The median length of hospital stay was 8 (range 4-60) days. Postoperatively, ten patients had high stoma output, six had ileus, two had anastomotic leak, one had non-ST elevation myocardial infarction, one had pneumonia, one had acute retention of urine, one had small bowel obstruction, 
Table II. Pre- and postoperative TNM staging of patients $(n=117)$.

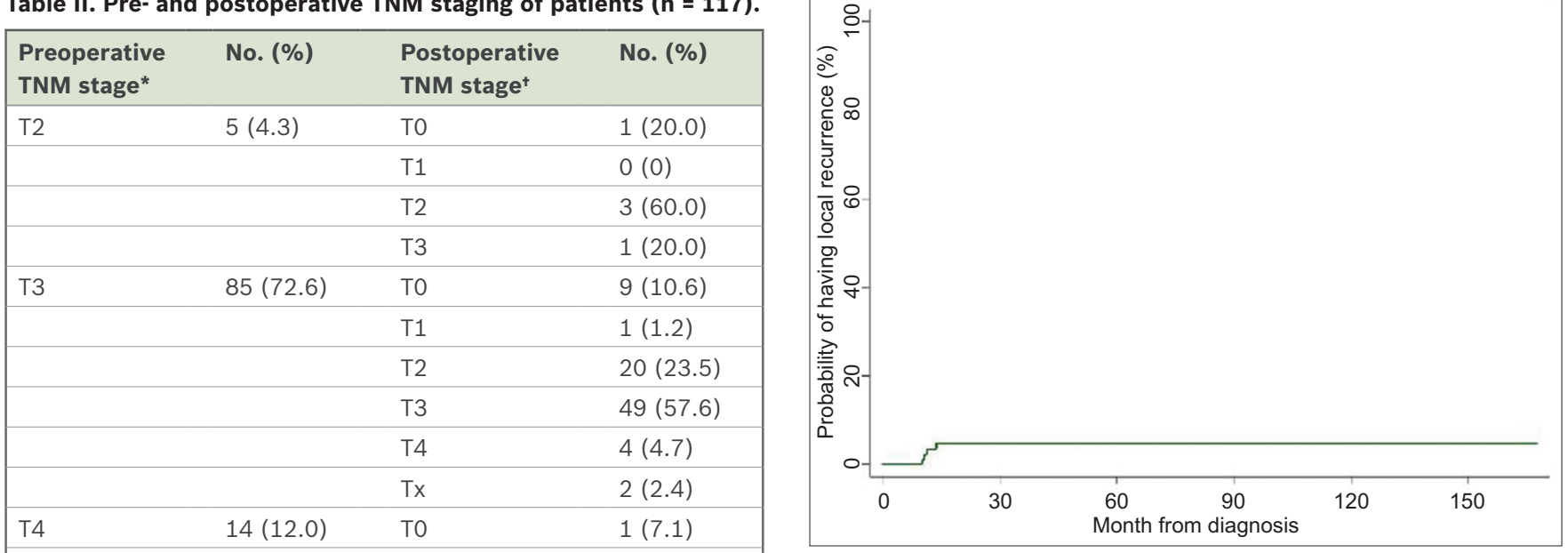

Fig. 1 Kaplan-Meier curve shows probability of local recurrence over time.

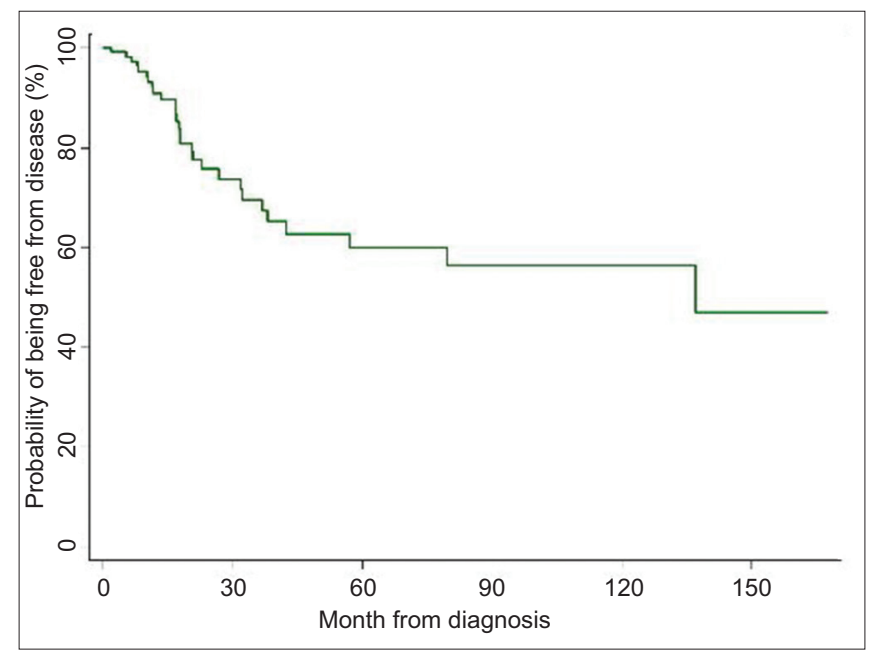

Fig. 2 Kaplan-Meier curve shows probability of disease-free survival over time.

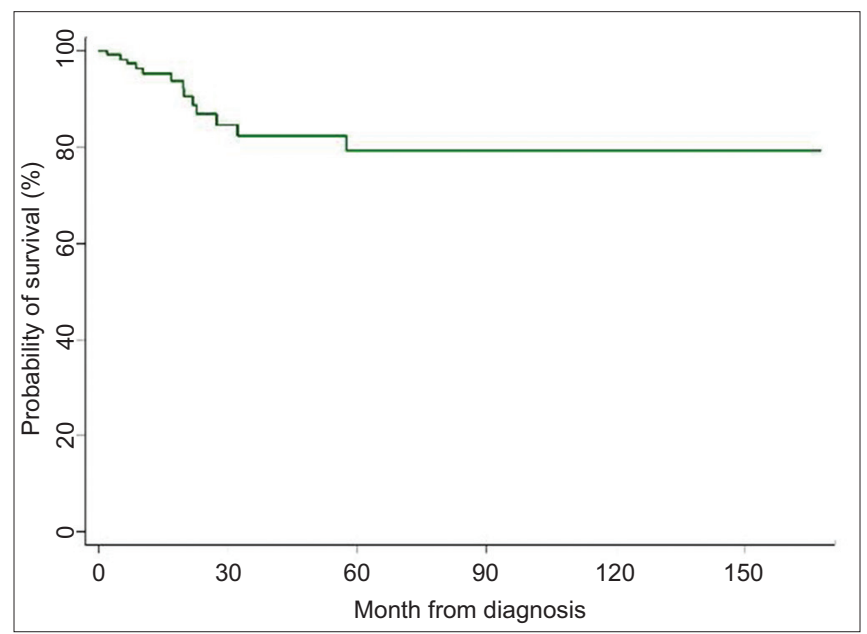

Fig. 3 Kaplan-Meier curve shows probability of overall survival over time.

pCR to treatment. For patients with T-stage tumours, $20.0 \%$ of CT2, $35.3 \%$ of $\mathrm{cT} 3$ and $64.2 \%$ of cT4 tumours were downstaged after neoadjuvant therapy. Among patients with $\mathrm{N}$-stage tumours, $55.6 \%$ of $\mathrm{cN} 1,90.0 \%$ of $\mathrm{cN} 2$ and $100.0 \%$ of $\mathrm{cN} 3$ tumours were downstaged.

In all, 23 patients were lost to follow-up during the study period. At the end of the study, four patients developed local

recurrence. The actuarial five-year local recurrence rate was $4.5 \%$ (Fig. 1). 31 patients developed distant metastasis and 19 died by the end of the study period. The five-year actuarial diseasefree survival and overall survival rates were $65.7 \%$ and $80.6 \%$, respectively (Figs. $2 \& 3$ ). 
Table III. Results of univariate Cox regression of potential factors affecting disease-free and overall survival.

\begin{tabular}{|c|c|c|c|c|}
\hline \multirow[t]{2}{*}{ Variable } & \multicolumn{2}{|c|}{ Disease-free survival } & \multicolumn{2}{|c|}{ Overall survival } \\
\hline & HR $(95 \% \mathrm{CI})$ & p-value & HR $(95 \% \mathrm{CI})$ & p-value \\
\hline Age at diagnosis & $0.97(0.94-1.00)$ & $0.028^{*}$ & $0.98(0.94-1.02)$ & 0.242 \\
\hline Gender & & 0.256 & & 0.649 \\
\hline Male & 1 (reference) & & 1 (reference) & \\
\hline Female & $1.49(0.75-2.94)$ & & $1.24(0.49-3.16)$ & \\
\hline Chemotherapy regimen* & & 0.568 & & 0.971 \\
\hline Oral & 1 (reference) & & 1 (reference) & \\
\hline Intravenous 5-FU & $1.24(0.60-2.56)$ & & $1.02(0.38-2.71)$ & \\
\hline Margin positivity ${ }^{+}$ & & 0.166 & & 0.488 \\
\hline Negative & 1 (reference) & & 1 (reference) & \\
\hline Positive & $1.89(0.77-4.64)$ & & $0.49(0.06-3.70)$ & \\
\hline Tumour downstaging $^{+}$ & & $0.029^{*}$ & & 0.462 \\
\hline No & 1 (reference) & & 1 (reference) & \\
\hline Yes & $2.37(1.09-5.14)$ & & $1.43(0.55-3.70)$ & \\
\hline Nodal positivity ${ }^{+}$ & & $0.005^{\ddagger}$ & & $0.009^{*}$ \\
\hline Positive & 1 (reference) & & 1 (reference) & \\
\hline Negative & $0.36(0.17-0.73)$ & & $0.28(0.11-0.72)$ & \\
\hline Pathological response $^{+}$ & & 0.224 & & 0.796 \\
\hline Complete & 1 (reference) & & 1 (reference) & \\
\hline Incomplete & $2.44(0.58-10.23)$ & & $1.21(0.28-5.30)$ & \\
\hline
\end{tabular}

*Excludes unknown chemotherapy status and private chemotherapy. +Excludes unknown values. $\neq p<0.05$ was statistically significant. 5-FU: 5-fluorouracil; $\mathrm{Cl}$ : confidence interval; HR: hazard ratio

Table IV. Results of multivariate Cox regression of potential factors affecting disease-free and overall survival.

\begin{tabular}{|c|c|c|c|c|}
\hline \multirow[t]{2}{*}{ Variable } & \multicolumn{2}{|c|}{ Disease-free survival } & \multicolumn{2}{|c|}{ Overall survival } \\
\hline & HR (95\% Cl) & p-value & HR (95\% CI) & p-value \\
\hline Age at diagnosis & $0.98(0.95-1.01)$ & 0.138 & $0.97(0.93-1.01)$ & 0.124 \\
\hline Gender & & 0.961 & & 0.806 \\
\hline Male & 1 (reference) & & 1 (reference) & \\
\hline Female & $0.98(0.46-2.10)$ & & $0.88(0.31-2.46)$ & \\
\hline Chemotherapy regimen* & & 0.201 & & 0.477 \\
\hline Oral & 1 (reference) & & 1 (reference) & \\
\hline Intravenous 5-FU & $1.69(0.76-3.78)$ & & $1.45(0.52-4.02)$ & \\
\hline Margin positivity ${ }^{+}$ & & 0.435 & & 0.349 \\
\hline Negative & 1 (reference) & & 1 (reference) & \\
\hline Positive & $1.45(0.57-3.67)$ & & $0.36(0.04-3.02)$ & \\
\hline Tumour downstaging ${ }^{+}$ & & 0.163 & & 0.764 \\
\hline No & 1 (reference) & & 1 (reference) & \\
\hline Yes & $1.86(0.78-4.42)$ & & $1.18(0.40-3.50)$ & \\
\hline Nodal positivity ${ }^{+}$ & & $0.006^{*}$ & & $0.009^{\neq}$ \\
\hline Positive & 1 (reference) & & 1 (reference) & \\
\hline Negative & $0.33(0.15-0.73)$ & & $0.26(0.10-0.72)$ & \\
\hline Pathological response ${ }^{+}$ & & 0.742 & & 0.982 \\
\hline Complete & 1 (reference) & & 1 (reference) & \\
\hline Incomplete & $1.32(0.26-6.74)$ & & $1.02(0.18-5.62)$ & \\
\hline
\end{tabular}

Adjusted for all variables listed in the table. *Excludes unknown chemotherapy status and private chemotherapy. + Excludes unknown values. $\neq p<0.05$ was statistically significant. 5-FU: 5-fluorouracil; Cl: confidence interval; HR: hazard ratio

Univariate and multivariate survival analysis of factors that might affect disease-free survival and overall survival rates showed that patients with positive nodes had significantly higher risks of poorer disease-free survival and poorer overall survival (Tables III \& IV). Age at diagnosis, gender, chemotherapy regimen, margin positivity, tumour downstaging and pCR did not predict worse disease-free survival or overall survival.

Acute and late treatment toxicities among our patients are shown in Table V. All patients completed the prescribed course of CRT; however only 96 patients had documented toxicities. 
Table V. Grade of acute and late toxicities associated with neoadjuvant chemoradiotherapy* $(n=96)$.

\begin{tabular}{|c|c|c|c|c|c|}
\hline \multirow[t]{2}{*}{ Toxicity } & \multicolumn{5}{|c|}{ Grade of toxicity [No. (\%)] } \\
\hline & 0 & 1 & 2 & 3 & 4 \\
\hline \multicolumn{6}{|l|}{ Acute } \\
\hline Nausea/vomiting & $87(90.6)$ & $7(7.3)$ & $2(2.1)$ & $0(0)$ & $0(0)$ \\
\hline Diarrhoea & $51(53.1)$ & $37(38.5)$ & $5(5.2)$ & $3(3.1)$ & $0(0)$ \\
\hline Cystitis & $91(94.8)$ & $4(4.2)$ & $1(1.0)$ & $0(0)$ & $0(0)$ \\
\hline Dermatitis & $31(32.3)$ & $37(38.5)$ & $26(27.1)$ & $2(2.1)$ & $0(0)$ \\
\hline Haematological & $94(97.9)$ & $1(1.0)$ & $1(1.0)$ & $0(0)$ & $0(0)$ \\
\hline \multicolumn{6}{|l|}{ Late } \\
\hline Gastrointestinal & $0(0)$ & $0(0)$ & $0(0)$ & $2(2.1)$ & $0(0)$ \\
\hline Genitourinary & $0(0)$ & $0(0)$ & $0(0)$ & $1(1.0)$ & $0(0)$ \\
\hline
\end{tabular}

*Multiple toxicities in the same patient were scored as separate events, and patients with unknown toxicity status were excluded from the calculation of percentages.

Among patients with known acute toxicities, 3 (3.1\%) patients had Grade 3 diarrhoea and 2 (2.1\%) had Grade 3 dermatitis. For late toxicities, 2 (2.1\%) patients had Grade 3 gastrointestinal toxicities (anastomotic fistula, $\mathrm{n}=1$; rectovaginal fistula, $\mathrm{n}=1)$ and $1(1.0 \%)$ had Grade 3 genitourinary toxicity (ureteric stricture). There were no Grade 4 toxicities.

\section{DISCUSSION}

Neoadjuvant CRT followed by surgery is the current standard of care for locally advanced rectal cancer. Using a preoperative approach for this study, we reported five-year local recurrence, disease-free survival and overall survival rates of about $5 \%$, $66 \%$ and $81 \%$, respectively. These results are comparable to published data. ${ }^{(2)}$

A pCR to preoperative treatment is an important outcome for rectal cancer. A pooled analysis of individual patient data showed that patients with locally advanced rectal cancer who achieved pCR after preoperative CRT had an improved five-year disease survival rate of $83.3 \%$ versus $65.6 \%$ for patients who did not achieve pCR. ${ }^{(7)}$ The pCR rate of $11.5 \%$ in our study was comparable to those of other published trials. ${ }^{(7,8)}$ However, our results showed that $\mathrm{pCR}$ after preoperative CRT did not improve disease-free survival or overall survival in our patients. In addition, tumour downstaging was not associated with improved outcomes. This was likely due to the small number of patients in our study.

One of the determinants of pathological downstaging is the interval from completion of CRT to surgery. Tulchinsky et al showed that an interval of more than seven weeks between completion of CRT and surgery improved $\mathrm{pCR}$ and disease-free survival in patients with rectal cancer. ${ }^{(9)}$ This was confirmed in a meta-analysis that showed that an interval to surgery of over eight weeks increased the pCR rate by $6 \% .{ }^{(10)}$ In our study, $53.8 \%$ (7/13) of patients who had surgery 12 weeks after the completion of CRT achieved pCR.

There are several studies that have looked at strategies to improve $\mathrm{pCR}$ rates. Firstly, escalation of radiotherapy dose in the preoperative setting has been shown to increase PCR rates. A recent Phase II study of a concomitant boost for IMRT-based neoadjuvant CRT in patients with Stage $2 / 3$ rectal cancer produced a high pCR rate of $23.7 \%$. ${ }^{(11)}$ Investigators at Utrecht University, the Netherlands, initiated a prospective trial investigating efficacy dose escalation using radiotherapy alone to a dose of 15 Gy $(5 \times 3$ Gy), delivered to the gross tumour volume prior to standard CRT. ${ }^{(12)}$ Secondly, preoperative CRT with combination chemotherapy has shown promise in increasing PCR rates. A small Taiwanese study reported an excellent pCR rate of $40 \%$ by using oxaliplatin and tegafur-uracil as part of the preoperative CRT regimen. ${ }^{13)}$ However, the optimal chemotherapy regimen delivered concurrently with radiotherapy remains to be established in randomised trials. Thirdly, researchers have investigated the delivery of chemotherapy in the interval period between completion of CRT and surgery. In a study by Habr-Gama et al, $65 \%$ of patients were found to have a pCR when additional chemotherapy was given during the resting period between neoadjuvant CRT and 'clinical' response assessment, ten weeks after the completion of radiation, with acceptable toxicity and high tolerability rates. ${ }^{(14)}$

Pathological nodal response after neoadjuvant chemoradiation is known to be associated with reduced local recurrence and improved survival rates. ${ }^{(15-17)}$ In our study, $38.5 \%$ of patients had nodal downstaging after preoperative CRT. However, this was not associated with reduced local recurrence or improved overall survival. Despite a meta-analysis demonstrating increased toxicity-related mortality risk ${ }^{(18)}$ with concomitant therapy, the treatment was well tolerated by our patients, with only $5.2 \%$ of patients having acute Grade 3 diarrhoea or dermatitis and 3.1\% of patients showing late toxicities.

Our study was limited by the relatively small number of patients studied compared to other larger landmark trials as well as the significant number of patients who were lost to followup. However, we were able to show that a treatment regimen comprising neoadjuvant CRT followed by TME surgery for locally advanced rectal cancer had comparable outcomes in our local population.

Currently, there is ongoing research in personalised patient treatment using predictive biomarkers to predict response to neoadjuvant therapy. These studies have the potential to change the way we manage patients. We await the outcomes. Meanwhile, the favourable outcomes of neoadjuvant CRT followed by TME surgery for locally advanced rectal cancer, in our institutional experience, support the continuation of this multimodality treatment for our local population. 


\section{REFERENCES}

1. National Registry of Diseases Office, Singapore. Singapore Cancer Registry Annual Registry Report Trends in Cancer Incidence 2014. Available at: https:// www.nrdo.gov.sg/docs/librariesprovider3/default-document-library/cancertrends-2010-2014_interim-annual-report_final-\%28public\%29.pdf?sfvrsn=0. Accessed February 20, 2016.

2. Sauer R, Becker H, Hohenberger W, et al; German Rectal Cancer Study Group Preoperative versus postoperative chemoradiotherapy for rectal cancer. N Engl J Med 2004; 351:1731-40.

3. Kapiteijn E, Marijnen CA, Nagtegaal ID, et al; Dutch Colorectal Cancer Group. Preoperative radiotherapy combined with total mesorectal excision for resectable rectal cancer. N Engl J Med 2001; 345:638-46.

4. Sauer R, Liersch T, Merkel S, et al. Preoperative versus postoperative chemoradiotherapy for locally advanced rectal cancer: results of the German CAO/ARO/AIO-94 randomized phase III trial after a median follow-up of 11 years. J Clin Oncol 2012; 30:1926-33.

5. Roh MS, Colangelo LH, $\mathrm{O}^{\prime}$ Connell MJ, et al. Preoperative multimodality therapy improves disease-free survival in patients with carcinoma of the rectum: NSABP R-03. J Clin Oncol 2009; 27:5124-30.

6. National Comprehensive Cancer Network. NCCN Clinical Practice Guidelines in Oncology (NCCN Guidelines $®$ ). Rectal Cancer. Version 3.2017. Available at: https://www.nccn.org/professionals/physician_gls/default.aspx. Accessed February 20, 2016.

7. Maas M, Nelemans PJ, Valentini V, et al. Long-term outcome in patients with a pathological complete response after chemoradiation for rectal cancer: a pooled analysis of individual patient data. Lancet Oncol 2010; 11:835-44.

8. Das P, Minsky BD. A watch-and-wait approach to the management of rectal cancer. Oncology (Williston Park) 2013; 27:962-8.

9. Tulchinsky H, Shmueli E, Figer A, Klausner JM, Rabau M. An interval > 7 weeks between neoadjuvant therapy and surgery improves pathologic complete response and disease-free survival in patients with locally advanced rectal cancer. Ann Surg Oncol 2008; 15:2661-7.
10. Petrelli F, Sgroi G, Sarti E, Barni S. Increasing the interval between neoadjuvant chemoradiotherapy and surgery in rectal cancer: a meta-analysis of published studies. Ann Surg 2016; 263:458-64.

11. Zhu J, Liu F, Gu W, et al. Concomitant boost IMRT-based neoadjuvant chemoradiotherapy for clinical stage II/III rectal adenocarcinoma: results of a phase II study. Radiat Oncol 2014; 9:70.

12. Burbach JP, Verkooijen HM, Intven $M$, et al. RandomizEd controlled trial for preoperAtive dose-escaLation BOOST in locally advanced rectal cancer (RECTAL BOOST study): study protocol for a randomized controlled trial. Trials 2015; 16:58.

13. Chao JY, Wang HM, Chiang FF, et al. Preoperative chemoradiotherapy with oxaliplatin and tegafur-uracil in locally advanced rectal cancer: pathologic complete response rate and preliminary results of overall and disease-free survival in a single institute in Taiwan. J Chin Med Assoc 2014; 77:128-32.

14. Habr-Gama A, Perez RO, Sabbaga J, et al. Increasing the rates of complete response to neoadjuvant chemoradiotherapy for distal rectal cancer: results of a prospective study using additional chemotherapy during the resting period. Dis Colon Rectum 2009; 52:1927-34.

15. Shivnani AT, Small W Jr, Stryker SJ, et al. Preoperative chemoradiation for rectal cancer: results of multimodality management and analysis of prognostic factors. Am J Surg 2007; 193:389-93; discussion 393-4.

16. Theodoropoulos G, Wise WE, Padmanabhan A, et al. T-level downstaging and complete pathologic response after preoperative chemoradiation for advanced rectal cancer result in decreased recurrence and improved disease-free survival. Dis Colon Rectum 2002; 45:895-903.

17. Valentini V, Coco C, Picciocchi A, et al. Does downstaging predict improved outcome after preoperative chemoradiation for extraperitoneal locally advanced rectal cancer? A long-term analysis of 165 patients. Int J Radiat Oncol Biol Phys 2002; 53:664-74

18. Fiorica F, Cartei F, Licata A, et al. Can chemotherapy concomitantly delivered with radiotherapy improve survival of patients with resectable rectal cancer? A meta-analysis of literature data. Cancer Treat Rev 2010; 36:539-49. 